\title{
DIRECT OBSERVATIONS ON SOME MANX SUBLITTORAL ALGAE
}

\author{
By Joanna M. KaIN \\ Marine Biological Station, Port Erin, Isle of Man
}

(Text-figs. I and 2)

The recent spread of the free-diving technique has made direct contact with the sublittoral environment relatively simple. The advantages of this to the ecologist need not be stressed.

The rather more cumbersome and expensive standard diving method had had already been used by Gislén (1930), Kitching, Macan \& Gilson (I934), Kitching (1937, I94I), Zalokar (1942), Bursa, Wojtusiak \& Wojtusiak (I948) and Waern (1952). Drach (1948 $a, b, 1949$, I95I) was the first to exploit free diving for ecological purposes and was followed by Forster (1954, 1955, I958), Pérès \& Piccard (1949), Ernst (1955), Laborel \& Vacelet (1958), Knight-Jones \& Clifford Jones (1956) and Aleem (1956).

Compared with the littoral region, the sublittoral of the British coast has been necessarily neglected. A certain amount of information has been collected by mechanical means (Chapman, 1947; Walker, 1947; Lodge, 1954), and where the bottom is smooth and the algae attached to small stones or unattached (Burrows, I958) a grab can be as reliable as a diver. It is the detailed flora of the uneven rock surfaces which, with the exception of Kitching's admirable studies with a diving helmet, has defied description. Forster has included the more important plants in his studies, and Knight-Jones has made small collections.

It is clear that these studies are still in the descriptive stage. A certain amount of description of the flora must precede any attempt at distinguishing the factors. It is for this reason that the preliminary picture which has been obtained of the sublittoral algae of some parts of the south end of the Isle of Man is being presented here. This should be more than of local interest; it should give an indication of the type of vegetation which exists below low water on rocky coasts of Britain. A general qualitative description of the distribution of the more important algae with depth on the rocky parts of the coast was all that was attempted at first.

The nomenclature used is that given in the 'Preliminary Check List' (Parke, I953) and the corrections to it (Parke, 1956, 1957). Where reliable taxonomic information was lacking, aggregates were used. Thus plants of Nitophyllum and Polyneura were grouped under Nitophyllum agg.; four-siphoned plants 
of Polysiphonia, other than P. elongata, were called P. urceolata agg. when ecorticate and $P$. violacea agg. when corticate.

\section{METHODS}

In order to cover as much ground as possible in this preliminary survey it was necessary to employ a sampling technique that was simple and rapid.

The depth was measured with a metre-long capillary tube gauge and expressed as metres below 'extreme low-water springs' (ELWS) by allowing for the height of the tide at the time of the dive. This was calculated in advance, using the data given by the Admiralty Tide Tables and Southward (1953), and corrected for deviation from the predicted height from observation of the Port Erin tide pole.

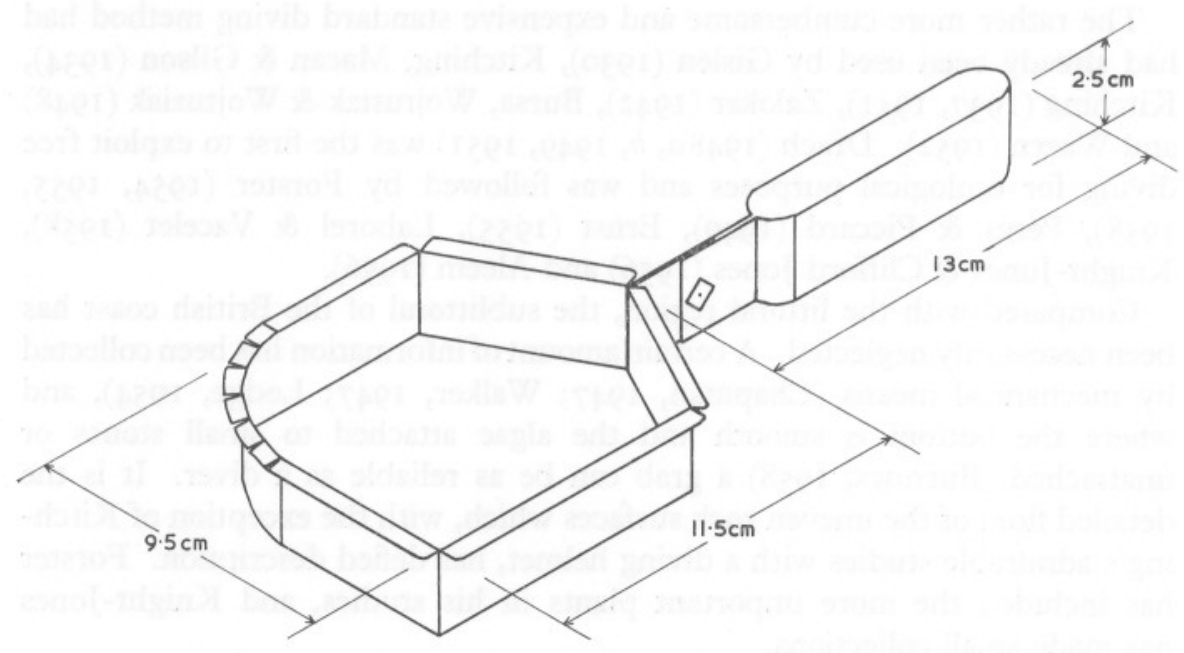

Fig. I. Diagram of the scraper used, without the net bag.

The algae were removed from the rock with the aid of a scraper (Fig. I), shaped somewhat like a miniature dredge. This was made from strip iron, had the lower leading edges sharpened, and bore a flange around the upper edges. A bag made from mosquito netting and measuring $40 \mathrm{~cm}$ in circumference and $66 \mathrm{~cm}$ in length had its neck drawn in by elastic cord. This fitted over the flange on the scraper and could easily be removed and replaced under water. The net had a wooden clothes peg attached near the neck and a roughened plastic label. The sharpened edge of the scraper easily removed the bases of most algae, except encrusting calcareous forms and large laminarians. The thalli so released passed into the net bag and were held there by the water passing through as the instrument was moved. 
A rapid survey of a chosen place was made by collecting samples of the flora from several rock faces at intervals of approximately I $\mathrm{m}$ of depth from ELWS to the lowest level of the rock itself. The net bag on the scraper was changed between each sample, the depth and substratum noted on the label and the bag attached by means of the clothes peg to a carrier. Each sample was examined in the laboratory and the species listed with notes on reproductive structures and a subjective abundance score. An exhaustive search for species was not made. Lithothamnion and related genera were ignored.

To show how far results were repeatable six levels at Port Erin breakwater were sampled twice within a few days. The general picture given of the distribution of the main species was similar in the two surveys, but the danger of attaching too much importance to the abundance scoring was emphasized by the apparent commonness of one species (Delesseria sanguinea) in one survey and its rarity in the other. Most of the places were sampled at one season of the year only, but Port Erin breakwater was sampled at regular intervals for one year for an indication of seasonal changes.

\section{THE REGION}

The places sampled all lie off the south-west end of the Isle of Man between Niarbyl and Langness (Fig. 2). This part of the coast is mainly rocky, with sand or shingle in the bays of Fleshwick, Port Erin, Perwick, Port St Mary, and Castletown. The sublittoral flora of these bays, where the bottom is relatively flat, forms a study in itself (Burrows, 1958) and has not been included here. Along much of the rest of the coast there are cliffs, varying in height and steepness. Below these sublittorally there are usually boulders, varying in size and extent with the topography of the cliffs. Opposite gullies the boulders have accumulated; below sheer cliffs they are fewer and more steeply stacked. In no place so far visited, however, do vertical cliffs extend for more than a few metres below ELWS; the slope of the rock is always more gentle. In certain places there are outcrops of solid rock either amongst the boulders or offshore from them. These form reefs of various shapes. The rocky bottom, whether formed of solid rock or boulders, always gives way at a depth of 8-21 m (mainly at about $12 \mathrm{~m}$ ) below ELWS to an unstable bottom of gravel or sand. Where the boulder bed slopes gently this change is gradual, there being gravel between the boulders for some distance before the gravel bed itself.

The principal factors likely to be important in the sublittoral environment will be briefly reviewed. They are, of course, interrelated.

The temperature of the sea varies between about $6^{\circ}$ and $15^{\circ} \mathrm{C}$ (Slinn, 1959). Except occasionally in the sheltered bays, and on rare occasions offshore in unusual weather conditions, there is little temperature stratification in this region. Taken by itself, therefore, temperature cannot be a factor determining the position of sublittoral species. The same applies to the concentrations of the major nutrients.

The substratum, on the other hand, is an extremely important factor in determining the algal distribution. As has been seen, the extent of rock surface suitable for colonization is very limited; much of the bottom is occupied by gravel. This latter may on occasion provide a suitable substratum for certain forms, but it seems likely that for much of the year not only is it uninhabitable itself, but its presence may reduce the suitability of some parts of the rock surfaces. The general topography of the rocks 
is important, the slope and aspect clearly affect the light intensity received, and may also have a bearing on the possible effect of the settling or scouring by sand or gravel. The type of surface does not vary a great deal and does not seem greatly to affect colonization.

It is likely that water movements affect algal distribution in a variety of ways. The tidal streams are in some places fairly strong, reaching 5 knots at springs in the vicinity of Calf Sound, and in other places are negligible. They may have some effect on

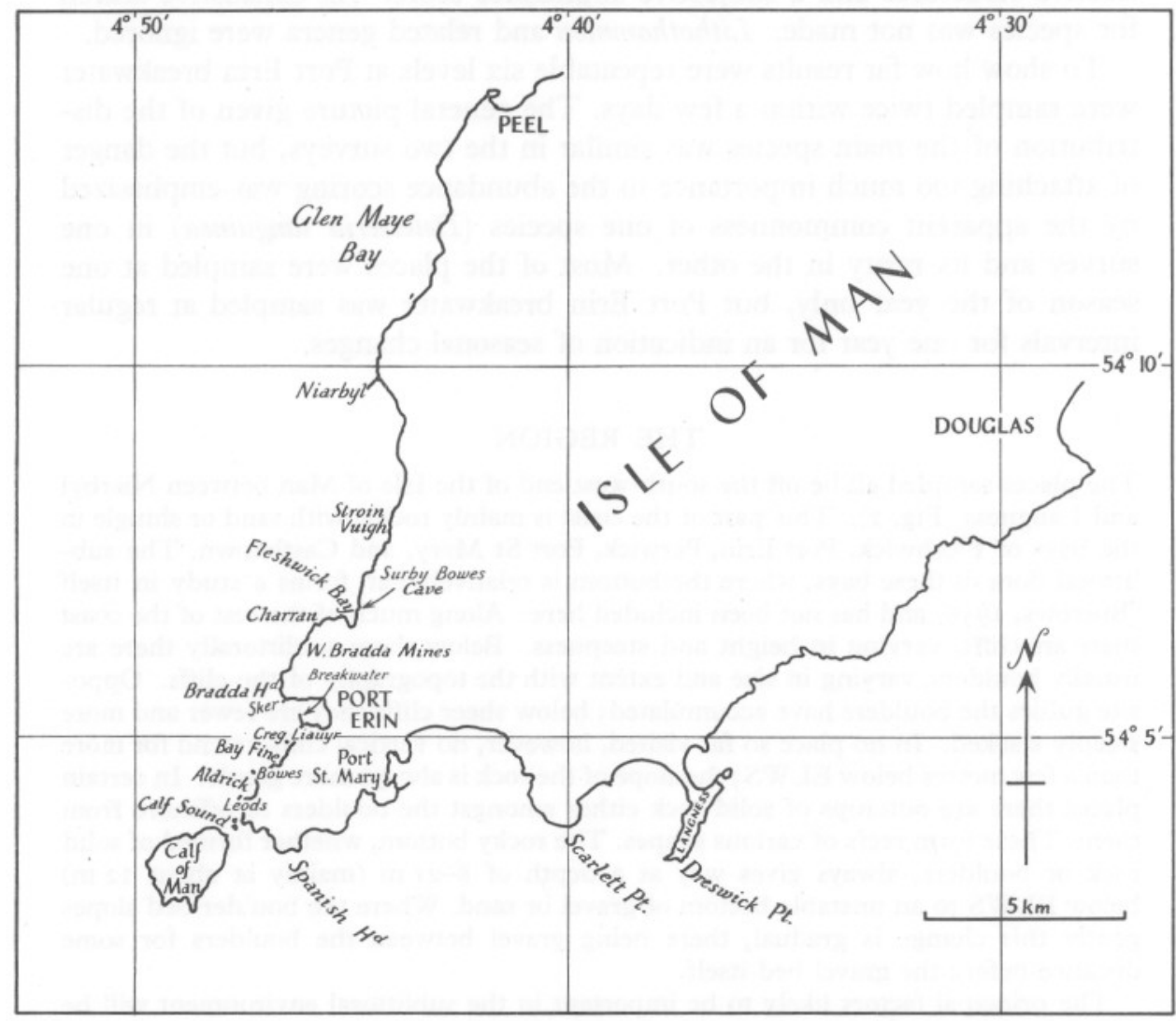

Fig. 2. Outline map of the south end of the Isle of Man.

the distribution of spores, and where they are strong growth may be affected through the provision of nutrient. The turbidity of the water and thus the light penetration is slightly increased at spring tides, but there is not the marked variation in underwater visibility that is so noticeable on other coasts.

Water movement of what is probably a more important type is caused by wave action. This is a notoriously difficult factor to measure on the shore and it is not any easier sublittorally. Its importance for a considerable depth below ELWS is beyond doubt during gales, as large quantities of sublittoral vegetation are washed on shore. It is possible that under more extreme conditions the water motion and the movement 
of solid material could be very considerable in the depth range under consideration. The movement of solid material could have indirect effects both on water turbidity and thus light penetration, and possibly through scouring action or settling on rock surfaces. The effects of gales on turbidity is just noticeable subjectively, but is not marked because there is no reservoir of suspendable material in the form of shallow water mud beds.

Light is clearly an important factor affecting algal distribution with depth. Direct measurements in these waters have only just begun and cannot yet be presented. It must now suffice to say that compared with many British waters the transmission is high and the compensation point for many algae subsequently deep. During the day there is sufficient light to see clearly at $35 \mathrm{~m}$ in summer, though of course it is virtually monochromatic.

\section{ROCKY BOTTOMS}

The places that have been studied will be described in turn around the coast. The records for all the species are given in Tables $\mathrm{I}-3$.

\section{Niarbyl}

At Niarbyl there is a conspicuous reef extending south-westwards from the shore, much of it above high-water level. The sublittoral part of the reef extends farther south-westwards and is highly dissected, there being a number of knolls with nearly vertical sides, about $8 \mathrm{~m}$ high, set on a smooth rock plateau at about Io $\mathrm{m}$ below ELWS. This plateau extends farther south-westwards for at least $100 \mathrm{~m}$, gently sloping and reaching a depth of $16 \mathrm{~m}$. On the lower parts gravel lies in slight fissures, and most of the surface is occupied by an ophiuroid bed. The point is subjected to fairly strong tidal streams.

The algae were sampled in May 1958 (Table I). The vertical and upward-facing slopes of the rocky knolls were occupied by typical laminarian forest, with Laminaria hyperborea dominant. An undergrowth to this was formed by Dictyota dichotoma (particularly abundant here), Desmarestia aculeata, Cryptopleura ramosa, Delesseria sanguinea, Cutleria multifida sporophyte and other species in smaller quantities. No Callophyllis laciniata was found here, though it was abundant in the forest farther south. Alaria esculenta occurred in places near the tops. The overhanging faces of the knolls bore few algae and were mainly occupied by the coelenterate Alcyonium. The zone of Laminaria had its lower limit at about 6-7 m below ELWS, leaving a gap of at least $2 \mathrm{~m}$ above the horizontal rock plateau. Most of the other common algae extended down through this region and also occupied much of the plateau. Saccorhiza polyschides, common in the lower parts of the Laminaria zone, tended to dominate just below it, both on the vertical rock faces and on the shallowest parts of the plateau at about $10 \mathrm{~m}$. Dictyota was also abundant in these lower parts, together with large bushes of Desmarestia. The vegetation gradually thinned out along the lower slopes of the plateau and was very sparse at the outer end of the reef.

\section{Stroin Vuigh}

A ridge of boulders runs out from the shore between Niarbyl Bay and Fleshwick Bay. The solid rock of the shore itself extends to about $7 \mathrm{~m}$ below ELWS, with occasional knolls. The boulders are numerous at this level but thin out gradually farther offshore, until they are only occasional at Io m, imbedded in fairly fine gravel. There is little or no tidal stream along this part of the coast.

The algae were sampled in July I959 (Table I). The usual Laminaria hyperborea 
TABLE 1. THE SPECIES OCCURRING IN SAMPLES FROM ROCKY PLACES BETWEEN NIARBYL AND BAY FINE, AT VARIOUS DEPTHS

(+ - present; $\mathrm{T}=$ tetrasporangia; $\mathrm{C}$ - cystocarps; $\mathrm{B}=$ both tetrasporangia and cystocarps; $\mathrm{P}=$ plurilocular sporangia; $\mathrm{p}=$ propagules.)

\begin{tabular}{|c|c|c|c|c|c|c|c|c|c|c|c|c|c|c|c|c|c|c|c|c|c|c|c|c|c|c|c|c|c|c|c|c|c|c|c|c|}
\hline \multirow[b]{2}{*}{ Depth (m below ELWS) } & \multicolumn{9}{|c|}{$\begin{array}{c}\text { Niarbyl } \\
\text { May } 1958\end{array}$} & \multicolumn{2}{|c|}{$\begin{array}{l}\text { Stroin } \\
\text { Vuigh, } \\
\text { July } \\
\text { 1959 }\end{array}$} & \multicolumn{2}{|c|}{$\begin{array}{c}\text { Surby } \\
\text { Bowes } \\
\text { Cave, } \\
\text { Oct. } \\
\text { I959 }\end{array}$} & \multicolumn{3}{|c|}{$\begin{array}{l}\text { Sker, } \\
\text { May } \\
\text { 1959 }\end{array}$} & \multicolumn{10}{|c|}{$\begin{array}{c}\text { Creg Liauyr }(\mathrm{N}), \\
\text { Apr. } 1958\end{array}$} & \multicolumn{10}{|c|}{$\begin{array}{c}\text { Creg Liauyr (S), } \\
\text { Apr. 1958 }\end{array}$} \\
\hline & I & 2 & 3 & 45 & 6 & 7 & 9 & II & 13 & 5 & 67 & o & I & 6 & 91 & II & 2 & 3 & 4 & $5 t$ & 67 & 78 & 9 & 10 & II 1 & 12 & I 2 & 3 & 4 & 5 & 6 & 78 & 89 & 10 & II & 12 \\
\hline haetomorpha meiagonium & + & - & + & - + & - & + & - & - & - & - & - - & - & - & - & - & - & - & - & - & - & - - & - - & - & - & - & - & + & - - & - & - & - & - & - - & - & - & - \\
\hline tuca & - & - & - & - & - & - & - & - & - & - & - - & - & - & - & - & - & - & - & - & - & - & - - & - & - & - & - & ++ & ++ & - & - & - & - - & - - & $-\quad-$ & - & - \\
\hline 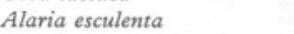 & + & - & - & +- & - & - & - & - & - & - & - - & - & - & - & - & - & 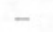 & - & - & - & - - & - - & - & - & - & - & : & +- & - & - & - & - & - - & - & - & \\
\hline$s a^{\star}$ & - & + & - & + & + & - & - & - & - & - & - - & - & - & - & - & - & - & - & - & - & - & - - & - & - & - & - & - & - - & - & - & - & - + & + & - & - & - \\
\hline sporoph. & - & + & + & ++ & + & + & + & + & + & + & ++ & - & - & ? & ? & $?$ & + & + & + & + & $+\quad+$ & ++ & + & + & + & + & -+ & ++ & + & + & + & ++ & ++ & + & + & + \\
\hline & 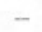 & + & - & ++ & + & - & + & + & - & 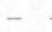 & $-\quad+$ & - & - & & + & + & 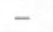 & - & + & - & $+\quad+$ & ++ & + & + & - & - & - & $+\quad-$ & + & - & + & ++ & $+\quad+$ & + & - & \\
\hline & 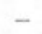 & - & - & - & - & - & - & - & - & - & $-\quad-$ & - & - & + & - & - & - & - & + & - & $-\quad-$ & - - & - & - & - & - & $-\quad$ & - & - & - & - & - & $-\quad-$ & $-\quad-$ & - & - \\
\hline chotoma & - & + & + & ++ & + & + & + & + & + & + & $+\mathrm{T}$ & - & - & + & + & + & + & + & + & + & $+\quad+$ & ++ & + & + & - & - & -+ & ++ & + & + & + & ++ & $+\quad-$ & $-\quad-$ & - & - \\
\hline to & - & - & - & -1 & - & - & - & - & - & & $-\quad-$ & - & - & - & - & - & - & - & - & - & $-\quad-$ & $-\quad-$ & - & - & - &.- & $-\quad-$ & $-\quad-$ & - & - & - & -+ & $+\quad-$ & $-\quad-$ & - & - \\
\hline tfo & 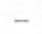 & - & - & - & - & - & - & - & - & - & $-\quad-$ & - & - & - & - & - & - & - & - & - & - - & $-\quad P$ & - & - & - & - & - & - & - & - & - & + & $-\quad-$ & -- & - & - \\
\hline . & 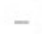 & - & - & - & - & - & + & + & - & - & $-\quad+$ & - & - & + & - & - & _- & - & - & - & $-\quad-$ & $-\quad-$ & - & - & - & - & - & - & + & - & - & - & $-\quad-$ & $-\quad-$ & - & - \\
\hline$m$ & & + & + & + & - & - & - & - & - & & +- & - & - & + & - & - & 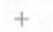 & 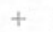 & + & +-1 & $+\quad+$ & $+\quad-$ & - & - & - & - & $\perp$ & $+\quad+$ & 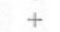 & + & + & - & - - & $-\quad-$ & - & - \\
\hline sa & 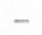 & - & - & $-\quad$ & - & - & - & - & - & - & $-\quad-$ & - & - & - & - & + & - & - & - & - & $-\quad-$ & $-\quad-$ & - & - & - & - & + & - & - & - & - & - & $-\quad-$ & $-\quad-$ & - & - \\
\hline vlai & 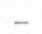 & - & - & - & - & - & - & - & 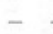 & 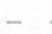 & - & - & - & - & - & - & - & - & - & - & $-\quad-$ & $-\quad-$ & - & -- & - & - & + & - & - & - & - & $-\quad-$ & $-\quad-$ & $-\quad-$ & - & \\
\hline 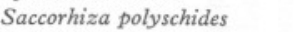 & & + & + & + & + & + & + & - & . & & $-\quad+$ & - & - & & + & - & . & 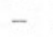 & - & - & $-\quad-$ & - & - & - & - & - & + & - & 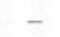 & + & + & ++ & $+\quad-$ & $-\quad-$ & - & \\
\hline Phac & - & - & - & - & - & - & - & - & - & - & - & - & - & - & - & - & - & - & - & - & $-\quad-$ & -+ & - & - & - & - & - & - & - & - & - & - & $-\quad-$ & $-\quad-$ & - & - \\
\hline plu & . & - & - & - & - & - & p & p & - & . & $-\quad-$ & - & - & - & - & - & - & - & - & - & $-\quad-$ & $-\quad-$ & - & - & - & - & $-\quad-$ & - & - & - & - & $\mathrm{p}$ & $-\quad-$ & $-\quad-$ & - & \\
\hline non & & - & - & - & . & 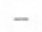 & & & 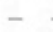 & & - & - & 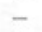 & & - & - & & & - & - & $-\quad-$ & $-\quad-$ & - & - & - & - & $-\quad-$ & - & 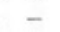 & - & - & - & - & $-\quad-$ & - & \\
\hline og & - & - & - & - & - & - & - & - & - & - & $-\quad-$ & - & + & - & - & - & - & - & - & - & $-\quad-$ & $-\quad-$ & - & - & - & - & - & $+\quad-$ & + & + & - & - & $-\quad-$ & -- & - & - \\
\hline$n n$ & - & - & - & $-\quad+$ & - & - & - & - & + & - & $-\quad-$ & - & - & - & - & - & - & - & - & - & $-\quad-$ & $-\quad-$ & - & - & - & - & $-\quad-$ & $-\quad-$ & - & - & - & - & - & $-\quad-$ & - & \\
\hline ha & . & - & - & - & - & - & - & - & - & - & $-\quad-$ & - & - & - & - & - & - & - & - & - & $-\quad-$ & -+ & - & - & - & - & $-\quad-$ & - & - & - & - & +- & - & -+ & + & \\
\hline ons & - & + & - & - & + & + & + & + & + & $\mathrm{T}$ & $-T$ & - & - & + & - & + & - & - & - . & - & - - & -+ & - & - & - & - & - & $-\quad-$ & - & - & - & -+ & +- & - & - & - \\
\hline okeri & - & - & - & $-\quad-$ & - & - & - & - & - & - & - & - & - & - & - & - & - & - & - & - & - $\quad-$ & $-\quad-$ & - & - & - & - & $-\quad-$ & - & - & - & - & $-\quad$ & $-\quad-$ & - & + & - \\
\hline & 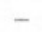 & - & - & - & - & - & - & - & - & - & - & - & - & - & - & - & + & & & & +- & -+ & - & - & - & & & + & - & + & & $-\quad-$ & - & $-\quad-$ & - & - \\
\hline$m$ agg. & & & - & - & - & & & - & - & & & - & & & & & & & & & & & & & & & & & & & & & & & & \\
\hline
\end{tabular}


Chondrus crispus

Chylocladia verticillata

Cruoria pellita

Cryptopleura ramosa

Cystoclonium purpureum

Delesseria sanguinea

Griffithsia flosculosa

Halarachnion ligulatum

Heterosiphonia plumosa

Hypoglossum woodwardit

Lomentaria clavellosa

L. orcadensis

Membranoptera alato

Nitophyllum agg.

Odonthalia dentat

Phycodrys rubens

Phyllophora rubens

Plocamium coccineum

Polysiphonia elongata

$P$. urecolata agg.

P. violacea agg.

Porphyropsis coccinea

Pterosiphonia parasitica

Pterosiphonia parasito plumosa

Ptilota plumosa

Rhodomela confervoides

Rhodophyllis divaricat

Rhodymenia palmata

Spermothamnion sp.

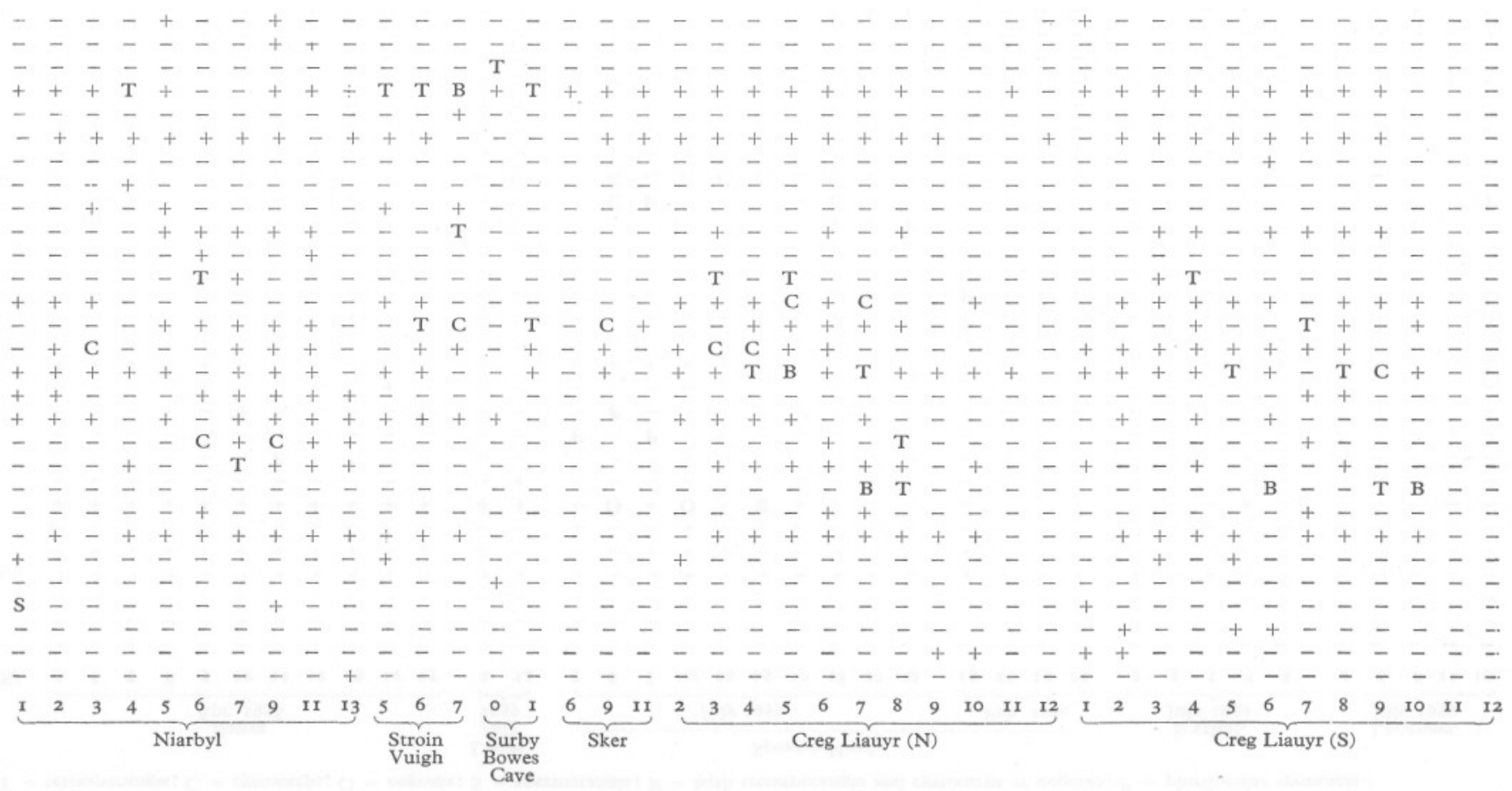

* Sensu Hamel, 1931-9.

† Sensu Irvine, 1956. 
TABLE 2. THE SPECIES OCCURRING IN SAMPLES FROM ROCKY PLACES BETWEEN BAY FINE AND LANGNESS, AT VARIOUS DEPTHS

$(+=$ present $; \mathrm{T}=$ tetrasporangia $; \mathrm{C}=$ cystocarps; $\mathrm{O}=$ oogonia; $\mathrm{S}=$ spermatangia $; \mathrm{B}=$ both tetrasporangia and cystocarps or oogonia; $\mathrm{P}=$ plurilocular sporangia. $)$

\begin{tabular}{|c|c|c|c|c|c|c|c|c|c|c|c|}
\hline \multirow[b]{2}{*}{ Depth (m. below ELWS) } & \multicolumn{11}{|c|}{$\begin{array}{c}\text { Bowes } \\
\text { Apr. 1958 }\end{array}$} \\
\hline & 5 & 6 & 7 & 8 & 9 & 10 & II & 12 & 13 & 14 & 15 \\
\hline Bryopsis plumosa & - & - & + & - & - & - & - & - & - & - & - \\
\hline Ulva lactuca & - & - & - & - & - & - & - & - & - & + & - \\
\hline Chorda tomentosa & - & - & - & - & + & - & - & - & - & - & - \\
\hline Cutleria multifida sporoph. & + & + & + & + & + & + & + & + & + & + & + \\
\hline Desmarestia aculeata & - & + & + & + & + & + & + & + & + & + & + \\
\hline . ligulata & - & - & - & - & - & - & - & - & - & - & - \\
\hline Dictyota dichotoma & + & + & + & + & + & + & + & + & + & + & + \\
\hline is membranacea & - & - & - & - & - & - & - & - & - & - & - \\
\hline s confervoides & - & - & - & - & - & - & $\mathbf{P}$ & - & - & - & - \\
\hline naldioides $\star$ & - & - & - & - & - & - & - & - & - & - & - \\
\hline ulosa $a^{\star}$ & - & - & - & - & - & + & - & - & - & - & - \\
\hline Halid & - & - & - & - & + & - & - & - & - & + & - \\
\hline erborea & + & + & + & + & + & + & - & - & - & - & - \\
\hline . sa & - & + & + & - & + & + & + & - & + & - & - \\
\hline$a c$ & - & - & - & - & + & - & + & + & + & - & - \\
\hline ia caespitula & - & - & - & - & - & - & - & - & - & - & - \\
\hline$S$ S l & - & - & - & - & - & - & - & - & - & + & 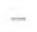 \\
\hline hon to & - & - & - & - & - & - & - & - & - & - & - \\
\hline Antithamnion plumula & - & - & - & - & - & - & - & - & - & - & - \\
\hline mo nation & - & - & + & + & - & - & - & - & - & - & - \\
\hline Bonn & + & - & + & - & + & + & + & + & + & - & - \\
\hline B. hamifera sporoph. & - & - & + & + & + & + & + & + & + & - & + \\
\hline artella byssoide & - & + & + & - & + & + & + & + & + & - & + \\
\hline Call & - & - & - & - & - & - & - & - & - & - & \\
\hline Callop & + & + & + & + & + & + & + & - & + & - & - \\
\hline Cerc & - & - & - & - & - & - & - & - & - & - & - \\
\hline Chond & - & - & - & - & - & - & - & - & - & - & - \\
\hline Comp & - & - & - & - & - & - & - & - & - & - & \\
\hline
\end{tabular}

\begin{tabular}{|c|c|c|c|c|c|c|c|c|c|c|c|c|c|c|c|}
\hline \multirow{2}{*}{\multicolumn{2}{|c|}{$\begin{array}{c}\text { Leods, } \\
\text { July } \\
\text { I959 }\end{array}$}} & \multicolumn{14}{|c|}{ Spanish Head, } \\
\hline & & \multicolumn{10}{|c|}{ July 1959} & \multicolumn{4}{|c|}{ Feb. 1960} \\
\hline 2 & 14 & 3 & 6 & 8 & 10 & 13 & 15 & 17 & 18 & 20 & 22 & 13 & 16 & 19 & 22 \\
\hline - & - & - & - & - & - & - & - & - & - & - & - & - & - & - & - \\
\hline - & - & - & - & - & - & - & - & - & - & - & - & - & - & - & - \\
\hline - & - & - & - & - & - & - & - & - & - & - & - & - & - & - & - \\
\hline+ & + & + & + & + & + & + & + & + & + & + & + & + & + & + & + \\
\hline . & - & - & + & + & + & + & - & - & - & - & - & + & - & - & - \\
\hline - & - & + & - & - & + & - & - & - & - & - & - & - & - & - & - \\
\hline+ & + & + & O & + & o & + & B & + & + & - & - & - & - & - & - \\
\hline- & + & - & - & - & - & - & - & - & - & - & - & - & - & - & - \\
\hline . & - & - & - & - & - & - & - & - & - & - & - & - & - & _- & - \\
\hline - & - & $\mathbf{P}$ & - & $\mathrm{P}$ & - & - & - & - & - & - & - & - & - & - & - \\
\hline - & - & - & $\mathbf{P}$ & - & - & - & - & - & - & - & - & - & - & - & - \\
\hline - & - & - & - & - & - & - & - & - & - & - & - & - & - & - & - \\
\hline 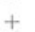 & - & + & + & + & + & + & - & - & - & - & - & + & . & . & - \\
\hline- & - & - & - & - & + & - & - & + & - & - & - & + & - & - & - \\
\hline - & - & + & - & + & + & + & + & + & + & - & - & + & + & . & - \\
\hline - & - & - & - & - & - & - & - & - & - & - & - & $\mathbf{P}$ & - & - & - \\
\hline - & - & - & - & - & - & - & - & - & - & - & - & - & - & - & - \\
\hline - & - & - & - & - & - & - & - & - & - & - & - & - & - & - & - \\
\hline - & - & - & - & - & - & - & - & - & $\mathrm{T}$ & + & $\mathrm{T}$ & - & - & + & - \\
\hline - & - & - & C & B & - & - & - & - & C & - & - & - & - & - & - \\
\hline . & C & - & C & C & C & C & C & C & C & C & - & - & - & - & - \\
\hline - & + & - & + & + & + & + & + & + & + & + & + & - & + & + & + \\
\hline - & - & - & - & - & + & - & + & - & - & - & - & - & - & - & - \\
\hline- & - & + & + & C & + & - & - & - & - & - & - & - & - & - & - \\
\hline+ & - & - & + & + & + & + & + & + & - & - & + & - & + & + & - \\
\hline - & - & - & - & - & - & - & - & - & - & - & - & - & - & - & - \\
\hline & - & - & - & - & - & - & - & - & - & - & - & - & - & - & - \\
\hline & + & - & - & - & - & - & - & + & $\mathrm{T}$ & $\mathrm{T}$ & $\mathrm{T}$ & - & - & - & - \\
\hline
\end{tabular}

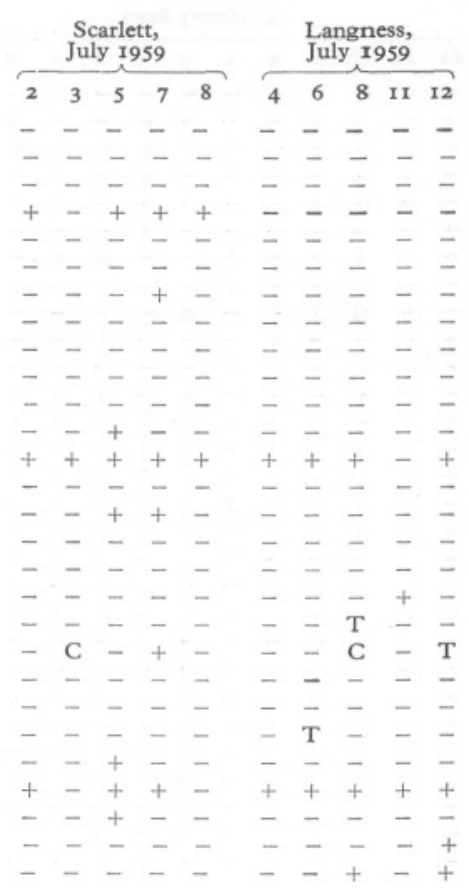


Corallina officinalis

Cruoria pellite

Cruoriopsis gracilis

Cryptopleura ramosa

Delesseria sanguinea

Dilsea carnosa

Griffithsia flosculosa

Halarachnion ligulatum

Halymenia latifolia

Heterosiphonia plumosa

Hypoglossum woodroardi

Lomentaria clavellosa

L. orcadensis

Membranoptera alata

Naccaria wiggii

Nitophyllum agg.

Odonthalia dentata

Phycodrys rubens

$P$. rubens

Plocamium coccineum

Polysiphonia elongata

P. urceolata agg.

$P$. violacea agg.

Porphyra umbilicalis $\mathrm{f}$. rosea

Porphyropsis coccinea

Pterosiphonia parasitica

Ptilota plumosa

Phodomela convervoides

Rhodochorton rothii (Turt.)

Näg.

Rhodophyllis divaricata

Rhodymenia palmata
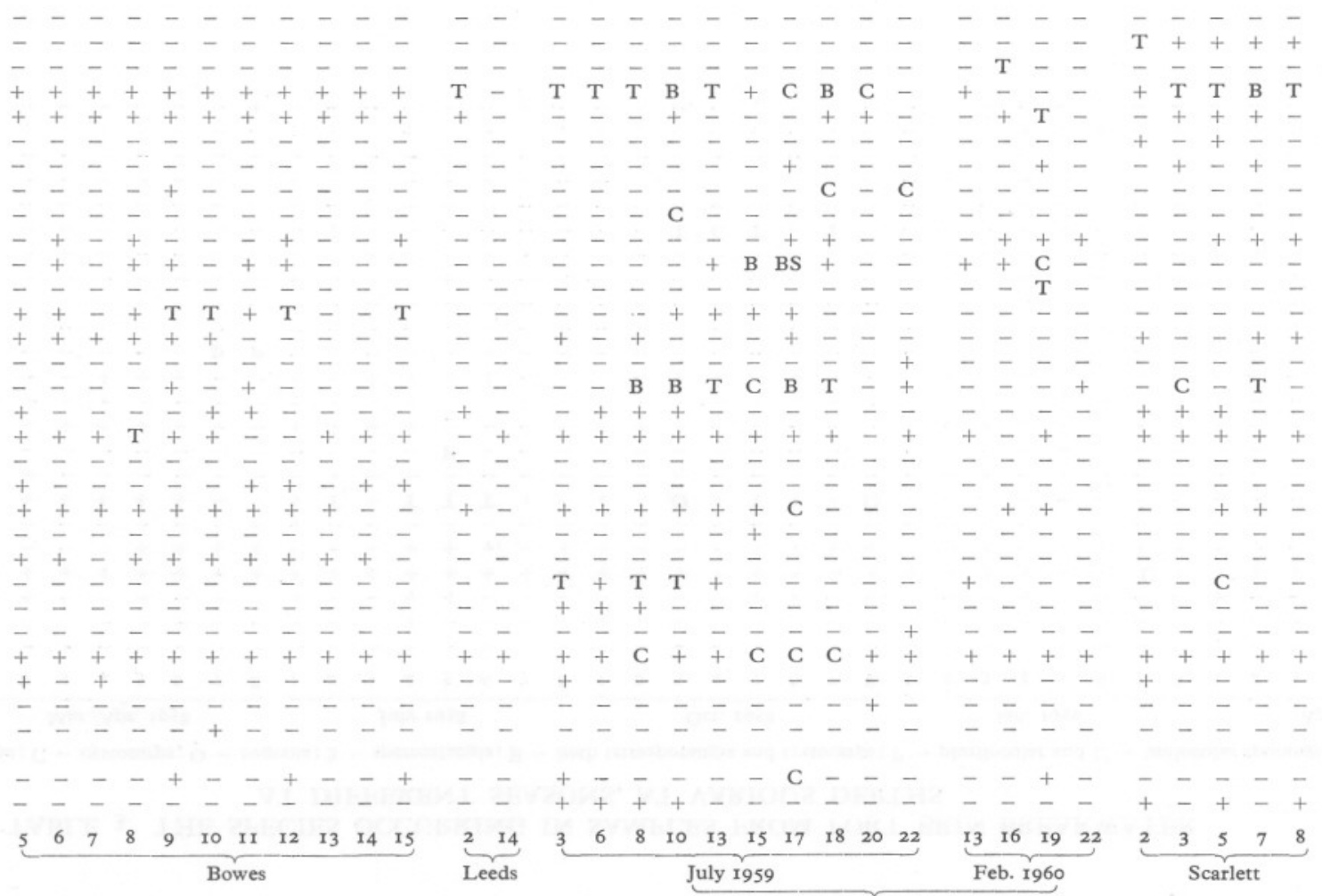

$-++$ Bowes

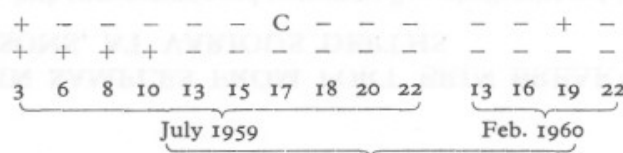

Scarlett 
TABLE 3. THE SPECIES OCCURRING IN SAMPLES FROM PORT ERIN BREAKWATER AT DIFFERENT SEASONS, AT VARIOUS DEPTHS

$(+=$ present $; \mathrm{T}=$ tetrasporangia $; \mathrm{C}=$ cystocarps; $\mathrm{O}=$ oogonia; $\mathrm{S}=$ spermatangia $; \mathrm{B}=$ both tetrasporangia and cystocarps; $\mathrm{P}=$ plurilocular and $\mathrm{U}=$ unilocular sporangia; $\mathrm{p}=$ propagules. $)$

\begin{tabular}{|c|c|c|c|c|c|c|c|c|c|c|c|c|c|c|c|c|c|c|c|c|c|c|c|c|c|c|c|c|c|c|c|c|c|c|c|c|c|c|c|c|}
\hline \multirow[b]{2}{*}{ Depth (m. below ELWS) } & \multicolumn{8}{|c|}{ Mar./Apr. 1958} & \multicolumn{8}{|c|}{ July r 958} & \multicolumn{8}{|c|}{ Oct. 1958} & \multicolumn{8}{|c|}{ Jan. 1959} & \multicolumn{8}{|c|}{ Apr. 1959} \\
\hline & I & 2 & 3 & 4 & 5 & 6 & 7 & 8 & I & 2 & 3 & 4 & 5 & 6 & 7 & 8 & $\mathrm{I}$ & 2 & 3 & 4 & 5 & 6 & 7 & 8 & I & 2 & 3 & 4 & 5 & 6 & 7 & 8 & $\mathbf{I}$ & 2 & 3 & 4 & 5 & 6 & 7 & 8 \\
\hline sis plumosa & - & - & - & - & - & - & - & - & - & - & - & - & - & - & - & - & - & - & - & - & - & - & - & - & - & - & - & - & - & - & - & - & + & - & - & - & + & + & t & - \\
\hline a melago & - & - & - & - & - & - & - & - & - & - & - & + & - & - & - & - & + & - & - & - & - & - & - & - & - & - & - & - & - & - & - & - & - & - & - & - & - & - & - & - \\
\hline lactuca & - & + & - & - & - & - & - & - & + & - & - & + & + & - & - & - & - & - & - & - & + & + & - & + & - & - & - & - & - & - & - & - & + & - & - & - & - & - & - & + \\
\hline tifida spor & - & + & + & + & + & + & + & + & + & + & + & + & + & + & + & + & + & + & + & + & + & + & + & + & + & + & + & + & + & + & $\mathrm{U}$ & + & - & + & + & + & + & + & + & + \\
\hline chenton & - & - & - & + & + & + & + & + & - & - & + & + & + & + & - & + & - & - & - & - & + & + & + & + & - & - & + & - & + & + & + & + & + & + & - & - & + & + & - & - \\
\hline lisulata & - & - & - & - & - & - & - & - & + & - & - & - & - & - & - & - & - & - & - & - & - & - & - & - & - & - & - & - & - & - & - & - & - & - & - & + & - & - & + & - \\
\hline otor & + & + & + & + & + & + & - & - & + & $T$ & + & $T$ & $\mathrm{~T}$ & $\mathrm{~T}$ & + & - & + & - & O & + & $\mathrm{T}$ & + & + & O & - & - & - & - & - & - & - & - & + & - & - & + & + & + & - & + \\
\hline gides & - & - & - & - & - & - & - & - & - & - & - & - & - & - & - & - & - & - & - & - & - & - & - & - & - & - & - & - & + & - & - & - & - & - & - & - & - & - & - & - \\
\hline 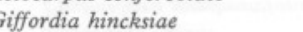 & - & - & - & - & - & - & - & - & - & - & - & - & $\mathbf{P}$ & - & - & - & - & - & - & - & - & - & - & - & - & - & - & - & - & - & - & - & - & - & - & $P$ & - & - & - & - \\
\hline orea & + & + & + & + & + & - & - & - & + & + & + & + & - & - & - & - & + & + & + & + & + & - & - & - & + & + & + & + & + & - & - & - & + & + & + & + & - & - & - & - \\
\hline acct & - & - & - & - & - & - & - & - & - & - & - & - & - & - & - & - & - & - & - & - & + & - & - & + & - & - & - & - & + & - & - & + & + & - & - & - & - & - & - & - \\
\hline ide & 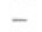 & - & - & + & + & + & - & - & + & + & + & + & + & + & - & - & - & - & + & + & + & + & - & - & - & + & - & + & + & - & - & - & + & + & - & + & + & - & + & - \\
\hline 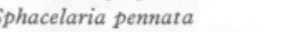 & - & - & - & - & - & - & p & 1 & 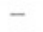 & - & - & - & - & - & - & - & - & - & - & - & - & - & - & - & - & - & - & - & - & - & - & - & - & - & - & - & - & $\mathrm{p}$ & - & - \\
\hline lum & - & - & - & - & - & - & - & - & - & - & - & - & - & - & - & - & - & - & - & - & - & - & - & - & - & - & - & - & - & - & - & - & - & - & - & - & + & - & - & $\mathrm{p}$ \\
\hline & - & - & - & - & - & - & - & 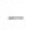 & 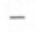 & - & - & - & - & - & - & - & - & 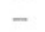 & - & - & - & - & - & - & - & - & - & - & - & - & - & - & - & - & + & - & - & - & - & \\
\hline & - & - & - & - & - & - & - & - & + & - & - & - & + & - & - & - & - & - & - & - & - & - & - & - & - & - & - & - & - & - & - & - & - & - & - & - & - & - & - & - \\
\hline thte & - & - & - & - & - & - & - & - & - & - & - & - & - & - & - & - & - & - & - & - & - & - & - & - & - & - & - & - & - & - & - & - & - & - & - & - & - & - & + & - \\
\hline & - & - & - & - & - & - & - & - & - & $T$ & - & - & - & - & - & - & - & - & $T$ & C & $\mathrm{T}$ & - & $T$ & - & - & - & - & - & - & - & + & - & - & - & - & - & - & - & - & - \\
\hline & - & - & - & - & - & - & - & - & - & 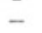 & - & - & - & - & - & - & - & - & - & - & - & - & - & - & - & - & - & - & - & - & - & - & - & - & - & - & - & - & + & - \\
\hline & - & - & - & - & - & - & - & - & - & - & - & - & - & - & - & - & - & - & - & - & - & - & - & - & - & - & - & - & - & - & - & - & - & - & - & - & + & - & + & + \\
\hline iide & - & - & - & - & - & - & - & - & - & - & - & - & - & - & - & - & - & - & - & - & - & - & - & - & - & - & - & - & - & - & - & - & - & - & - & - & - & + & + & - \\
\hline & + & + & + & - & - & - & - & - & + & + & - & + & - & - & - & + & + & + & + & + & + & 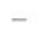 & + & - & + & + & - & + & + & + & - & + & + & + & + & 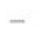 & - & - & - & - \\
\hline nium rubrum & - & - & - & - & - & - & - & + & - & - & - & - & - & - & - & - & - & - & - & - & & - & & & & & & & & - & - & & & & _- & - & & & & \\
\hline
\end{tabular}


Corallina officinalis Cruoria pellita

Cryptopleura ramosa

Delesseria sanguinea

Dilsea carnosa

Dumontia incrassata

Halarachnion ligulatum

Heterosiphonia plumosa

Hypoglossum woodwardit

Lomentaria clavellosa

L. orcadensis

Membranoptera alata

Nitophyllum agg.

Odonthalia dentata

Phycodrys rubens

Phyllophora rubens

Plocamium coccineum

Polysiphonia elongata

P. nigra

P. urceolata agg.

P. violacea agg.

Porphyrropsis coccinea

Pterosiphonia parasitica

Ptcrosiphonia parasilo plumosa

Ptilota plumosa
Rhodomela confervoides

Rhodophyllis divaricata

Rhodymenia palmata

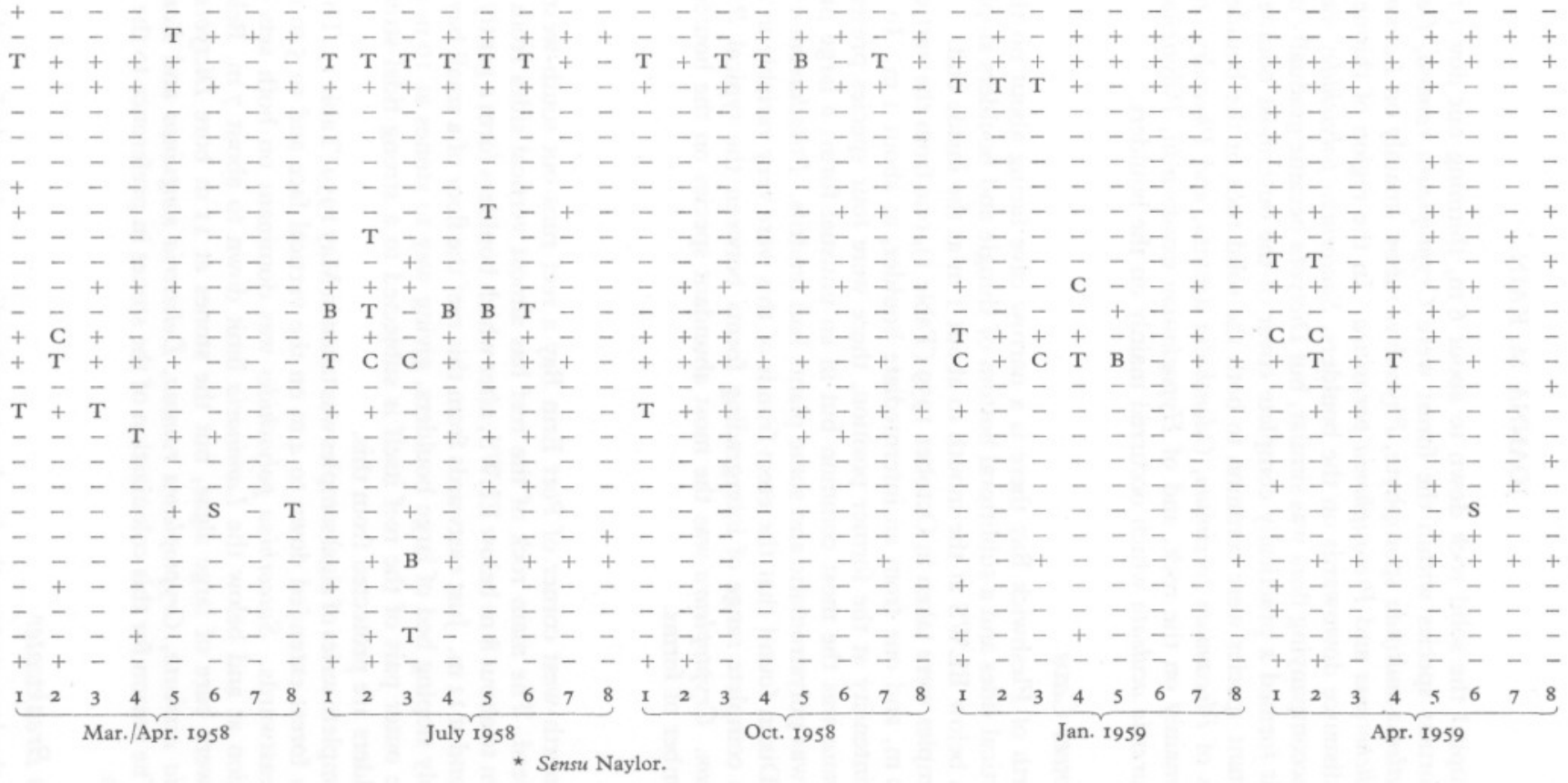


forest occupied the solid rock down to about $6 \mathrm{~m}$, thinning out down to $8 \mathrm{~m}$. The most important species within the forest were Cryptopleura ramosa, Delesseria sanguinea, Cutleria multifida sporophyte, Phycodrys rubens (mainly on Laminaria stipes), Dictyota dichotoma and Pterosiphonia parasitica. In the region of thinner forest and for some distance downwards on the boulders, Saccorhiza polyschides was dominant. Here the accompanying flora was similar, but Dictyota became gradually more abundant until it formed a practically complete cover of the outermost boulders. Most of the important species were common to both the solid rock or the boulders, with the exceptions of Plocamium coccinium, Odonthalia dentata, and Phycodrys rubens which occurred mainly on the rock, and of Hypoglossum woodwardii, Polysiphonia elongata and Desmarestia aculeata which occurred mainly on the boulders.

\section{Surby Bowes Cave}

Just north of Fleshwick Bay there is a narrow cave facing about north-west, with almost vertical sides and a sublittoral bottom of shingle and boulders sloping up from about $3 \mathrm{~m}$ below ELWS at the mouth to about $\mathrm{I} \mathrm{m}$ at the inside end.

Two samples were taken in October 1959 (Table I), one from the vertical end wall, at about $\mathrm{om}$, and one from an intermediate boulder, at about $\mathrm{I} \mathrm{m}$. In spite of the low light intensity at the former position, there were four species present. Cryptopleura ramosa was the most common but in an unusual form; a large proportion of the frond was polystromatic and some plants had tendrils. Ptilothamnion was present here: Dr Dixon found that the erect fronds of this were very variable in appearance, showing a complete range of intergrading forms between the typical $P$. pluma and $P$. lucifugum. Cryptopleura was the most abundant species on the boulder, where it took a number of forms.

\section{The Sker}

At the north-west corner of Port Erin Bay a reef runs out south-westwards from Bradda Head. The main rock of the reef has almost vertical sides from above high water down to about $8 \mathrm{~m}$ below ELWS, after which boulders form a gently sloping bed down to sand at II m. Just eastwards from this reef the floor of a small bay is occupied by a steeply sloping bed of large boulders, giving way to stones at $10 \mathrm{~m}$ and sand at II $\mathrm{m}$. The outer part of the reef itself is subjected to a strong tidal stream, but the inner boulders are protected from this.

An incomplete series of algal samples was taken in May 1959 (Table I). The Laminaria hyperborea forest extended down to $4 \mathrm{~m}$ on the vertical face and to $5 \mathrm{~m}$ on the large boulders eastwards. Saccorhiza polyschides was dominant on both sets of boulders in the region at and below the Laminaria limit down to about $7 \mathrm{~m}$. Below that the boulders were bare of large algae, but the stones at II m bore Dictyota dichotoma, Desmarestia aculeata, Cryptopleura ramosa, Delesseria sanguinea and Laminaria saccharina. The reason for this colonization of the stones in preference to the rocks is not yet certain.

\section{Port Erin Breakwater}

During the last century the breakwater extending northwards from the south-west corner of Port Erin Bay collapsed and the large concrete blocks and boulders from which it was built now form an uneven ridge with its top at about mid-tide, sloping steeply on the inside (east) to sand at about $7 \mathrm{~m}$ below ELWS and sloping gently on the outside to sand with gravel in places at $9 \mathrm{~m}$. The upper parts are formed only of 
concrete blocks about $2 \mathrm{~m}$ long, while the lower parts have boulders mixed with the blocks. Tidal streams are weak and effect only the top and end.

Sampling has been carried out at three-monthly intervals throughout a year on the outer (western) side. The records are given in Table 3. The Laminaria hyperborea forest was thick on the upper surface of the concrete blocks down to 5-6 m. At this level Saccorhiza polyschides dominated at all times of year. The most abundant smaller plants at all levels were Cryptopleura ramosa, Delesseria sanguinea, Dictyota dichotoma, Phycodrys rubens, Cutleria multifida sporophyte, Desmarestia aculeata and Odonthalia dentata. There was no marked change in the qualitative composition of the flora during the year, except for the apparently complete absence of Dictyota in January. That the disappearance of this species from sublittoral rocks in winter is a regular occurrence was confirmed the following year. The forms of many of the species showed a seasonal change, the fronds of such plants as Delesseria and Odonthalia becoming denuded in the autumn and regenerated in January. There was no suggestion of a summer annual flora. More species were recorded in April 1959 than April 1958; this may have been a reflexion of improved technique rather than a difference between the years.

\section{Creg Liauyr}

Between Port Erin breakwater and Bay Fine an exposed reef extends west-north-west from the shore. Beyond this, under water, there are a number of isolated rocky knolls with steep sides, surrounded by large boulders which slope steeply to the level of the gravel, at $13.5 \mathrm{~m}$, where they stop abruptly. Just south-west of the reef the boulders are smaller, $\mathrm{I}-2 \mathrm{~m}$ across, and slope more gently down to the gravel. The region is subjected to some tidal stream at springs but very little at neaps.

Two series of samples were taken, one ('north') on the rocky knolls and large boulders, and the other ('south') on the continuous boulder bed, in April-June 1958 (Table I). In both places there was the usual thick forest of Laminaria hyperborea which faded out between 6 and $8 \mathrm{~m}$ below ELWS. Again Saccorhiza polyschides was most abundant at this level. The undergrowth flora in both places consisted mainly of Phycodrys rubens (mostly on Laminaria stipes), Cryptopleura ramosa, Delesseria sanguinea, Dictyota dichotoma, Cutleria multifida sporophyte, Desmarestia aculeata, Odonthalia dentata, Pterosiphonia parasitica, Plocamium coccineum, Membranoptera alata and Callophyllis laciniata. Most of these extended below the Laminaria forest for about $2 \mathrm{~m}$ but the Cutleria sporophyte was the principal occupier (though not forming a continuous cover) of the lowest boulders at II-I2 m. There was little difference between the main constituents of the flora of the two regions, but the smaller boulders south showed a greater variety in the more occasional species.

\section{The Bowes}

Between Bay Fine and Aldrick a rocky reef lies just off the shore, and is known as Halfway Rocks or the Bowes. Part of this is visible at all stages of the tide, but offshore from it there is an extensive submerged rock with its pinnacle at $5 \mathrm{~m}$ below ELWS. The gravel bed lies deeper here than nearer Port Erin, with the result that there are rocky surfaces down to $16 \mathrm{~m}$ below ELWS. The reef itself is very uneven with ledges, cliffs and overhangs. The reef is subjected to fairly strong tidal streams even at neaps.

The main sampling series was made in April-May 1958 (Table 2). The Laminaria hyperborea was as dense as found in the previous places mentioned, and thinned out between 10 and $12 \mathrm{~m}$. Here again Saccorhiza polyschides formed a dominant zone at the lower limit of Laminaria. The associated flora was also similar. The similarity of the flora to that of the breakwater and Creg Liauyr is remarkable in that the whole 
spectrum is $4-5 \mathrm{~m}$ lower. The top of the Bowes rock is at the same level as the lower limit of Laminaria at the breakwater, but the forest is thick for another $5 \mathrm{~m}$ downwards. Some algae were present on the lowest rock surfaces at $16 \mathrm{~m}$, though the cover was sparse; the most common were Desmarestia aculeata, Delesseria sanguinea, Cutleria multifida sporophyte, and Heterosiphonia plumosa. In December the only occupant of this level had been Cutleria sporophyte.

\section{The Leods}

Near the entrance to Calf Sound a reef known as the Heifer Rocks or the Leods lies offshore with its top just above LWS. The main shore is steep at this point, with some boulders at the base. The channel between here and the reef is narrow with a rocky bottom at about Io m below ELWS. The side of this formed by the reef is slightly overhanging, while the south-western side of the reef is about vertical with a boulder bed at its base at $14 \mathrm{~m}$, sloping gently down to the gravel at $17 \mathrm{~m}$. The northern side of the reef is also a sloping boulder bed. The reef is subjected to strong tidal streams.

An incomplete series of samples was taken in July 1958 and I959 (Table 2). Laminaria hyperborea extended to 6-10 $\mathrm{m}$. The steep inner side of the reef was occupied by Alcyonium below about $3 \mathrm{~m}$ where it overhangs. The flora in the Laminaria forest had the usual composition, while the south-west boulders at $14 \mathrm{~m}$ were occupied mainly by Dictyopteris membranacea, Dictyota dichotoma, Cutleria multifida sporophyte, Bonnemaisonia asparagoides and B. hamifera sporophyte.

\section{Spanish Head}

Spanish Head lies on the other side of Calf Sound and consists of steep cliffs with large boulders ( $2-3 \mathrm{~m}$ across) below them under water. These slope steeply (at about I in 5) into deep water and provide the deepest rock so far encountered on the island at $22 \mathrm{~m}$ below ELWS. The Head is subjected to very strong tidal streams on both flood and ebb.

Sampling was first carried out in July 1959 (Table 2). The Laminaria hyperborea plants were large and the forest started thinning at about $9 \mathrm{~m}$ but extended to $\mathrm{I} 4 \mathrm{~m}$ below ELWS. Saccorhiza polyschides extended over a wide range, down to $\mathrm{I} 9 \mathrm{~m}$, but was again probably most abundant at the lower limit of Laminaria. The main species occurring within the forest and some distance below it were Cryptopleura ramosa, Phycodrys rubens, Plocamium coccineum, Cutleria multifida sporophyte, Pterosiphonia parasitica, Desmarestia aculeata, Dictyota dichotoma, Callophyllis laciniata and Nitophyllum agg. Those confined to above $10 \mathrm{~m}$ were Rhodymenia palmata, Callithamnion tetragonium and Porphyra umbilicalis f. rosea (all on Laminaria fronds), Desmarestia ligulata and Odonthalia dentata. Below ro $\mathrm{m}$ the vegetation became sparse and irregular; some of the boulders had a fair cover of small forms, some were bare and some were colonized by Alcyonium. From 1o to $18 \mathrm{~m}$ the most important species were Bonnemaisonia asparagoides, B. hamifera sporophyte and Hypoglossum woodwardii, in addition to those already mentioned. Below this the vegetation was very sparse indeed and a close search was necessary to detect it. Even so, there were still eleven species present on the lowest boulders at $22 \mathrm{~m}$, the most important being Antithamnion plumula, Bonnemaisonia hamifera sporophyte, Compsothamnion thuyoides, Pterosiphonia parasitica and Cutleria multifida sporophyte. The crinoid Antedon occupied many of the boulders.

In February 1960 further sampling was carried out on the lower boulders in order to determine whether the sparse algal cover was confined to the summer. Although the vegetation was considerably reduced in quantity the majority of the species were 
still present (Table 2). The most marked absence was of Dictyota dichotoma, which also disappeared from other sublittoral rocks in winter. Bonnemaisonia asparagoides and Compsothamnion thuyoides had also apparently disappeared. Although it was almost too dark to read at the lowest depth, at least five species were still present. Plocamium coccineum was abundant at $19 \mathrm{~m}$, where there was also a first year sporeling of Laminaria hyperborea (with mucilage canals in the stipe). This was the deepest record for this plant.

\section{Scarlett Point}

The shore just east of the southern tip of Scarlett Point is formed of limestone ledges, each ledge being inclined slightly upwards towards the sea. Sublittorally the configuration is very similar, though obscured by the thick forest of $L$. hyperborea. The general slope is very gentle and in places there are patches of gravel. There are also shallow gullies containing boulders.

An incomplete series of samples was taken in July 1959 (Table 2). The Laminaria forest was very thick. The rock below was noticeably bare, apart from a cover of Lithothamnion and small quantities of the usual species. Even Cutleria multifida sporophyte, the most regular species, was present in only very small amounts in the really thick forest at $\mathrm{I}-5 \mathrm{~m}$; its place was largely taken by Cruoria pellita. All these extended over the depth range sampled (the level of the gravel bed proper was not reached), except for Odonthalia dentata which ceased at $5 \mathrm{~m}$. The boulders in the gullies were occupied by Laminaria saccharina.

\section{Dreswick Point (Langness)}

Langness is a narrow peninsula extending southwards and Dreswick Point is the southern tip of this. Offshore the tidal stream is notoriously strong, but there is little evidence of this inshore. Just west of the lighthouse a gully runs southwards, with boulders on its gently sloping floor and several steep-sided knolls of rock about $8 \mathrm{~m}$ high.

An incomplete series of samples was taken from a knoll and the boulders in July I959 (Table 2). The knoll was occupied by $L$. hyperborea forest as usual, but the associated flora differed somewhat from that common near Port Erin: Heterosiphonia plumosa was the most prominent red alga, and neither Cutleria multifida sporophyte nor Dictyota dichotoma was recorded. The L. hyperborea extended to the bottom of the knoll, at II m below ELWS, with occasional plants on the boulders. There was no Saccorhiza polyschides zone, the boulders being occupied instead by L. saccharina. The level of the gravel bed was not reached, though there were patches of coarse sand amongst the boulders.

\section{UNSTABLE BOTTOMS}

Over the course of a number of years Lodge (1954) carried out a great many dredging operations around the south end of the Isle of Man and recorded a total of I 28 species. Many of these have not been found on the rock surfaces, and as some of the areas which she dredged were gravel beds it seems likely that a considerable flora could develop on the unstable bottom under certain conditions. Little attempt has been made to extend these particular observations using diving equipment, but the few direct records on gravel that have been made (Table 4) may be of interest. It must be emphasized that these algae were attached to superficial stones and were clearly not drifting. 


\section{Glen Maye Bay}

Glen Maye Bay, just north of Niarbyl, was sampled in July 1959. The bottom consists of small boulders and stones mixed with gravel. At II $\mathrm{m}$ below ELWS the gravel (diameter 2-30 $\mathrm{mm}$ ) bore Chorda filum, Brongniartella byssoides, Myriocladia? sp., Stictyosiphon tortilis, Cutleria multifida gametophyte (male and female), Sporochnus pedunculatus, Cladostephus spongiosus, Acrothrix gracilis and other species. This assemblage clearly differed from those found on the rock reefs. Brown algae were predominant. Further offshore, at $\mathrm{I} 2 \mathrm{~m}$, there were small boulders and these bore many of the species normally inhabiting rock surfaces: Saccorhiza polyschides, Dictyota dichotoma, Halidrys siliquosa, Heterosiphonia plumosa, Cutleria multifida sporophyte, Hypoglossum woodwardii, Brongniartella byssoides, Pterosiphonia parasitica and Phycodrys rubens being the most common.

\section{Stroin Vuigh}

In July 1959 the lowermost boulders ( $10 \mathrm{~m}$ ) at Stroin Vuigh, already described, bore a flora very similar to that on the Glen Maye boulders. In addition, the gravel between the boulders was occupied by Myriocladia? sp., Chorda filum, Enteromorpha clathrata and Cutleria multifida gametophyte. Myriocladia? sp. was also found by Parke (private communication) in this region and then ascribed by her to M. loveni J.Ag. This was later found to differ from the Swedish material of this species (Parke 1950). Its identity is not yet certain.

\section{Off Bradda Hill}

In September 1958 small stones at $15 \mathrm{~m}$ below ELWS off the Charran, west of Fleshwick Bay, were occupied by Brongniartella byssoides, Saccorhiza polyschides sporelings and Plocamium coccineum. At $2 \mathrm{I} \mathrm{m}$ the bottom was sand and gravel and there were no algae.

In August 1959 an extensive search was made at $32 \mathrm{~m}$ and at $23 \mathrm{~m}$ on the coarse gravel bed off the West Bradda Mines, but no attached algae were found. The calm sunny summer would have resulted in there being growth, given a spore supply, if this were ever possible, but clearly either the depth or tidal streams prevented it.

\section{Off Creg Liauyr}

Again in August 1959, somewhat shallower beds off Port Erin breakwater between Bradda Head and Bay Fine, where the tide is less strong, were found to support a fairly rich flora. The most abundant species, on stones of $5-20 \mathrm{~cm}$, mixed with coarse sand, were Saccorhiza polyschides, Stictyosiphon tortilis, Sporochnus pedunculatus, Arthrocladia villosa, Desmarestia aculeata, Cutleria multifida gametophyte, Bonnemaisonia asparagoides, Antithamnion plumula, Halarachnion ligulatum and Scinaia furcellata. The gravel at $13 \mathrm{~m}$ adjacent to the boulders at Creg Liauyr was found in September 1958 to bear Scinaia, Halarachnion, Antithamnion sarniense and Sporochnus pedunculatus.

The same position was visited during the winter, at the end of November 1959, and the flora found to be almost negligible. Clearly the intervening gales had destroyed most of the plants which were dependent on the unstable bottom not being extensively disturbed. 
TABLE 4. THE SPECIES OCCURRING IN SAMPLES FROM UNSTABLE BOTTOMS, AT VARIOUS DEPTHS

$(+=$ present $; \mathrm{T}=$ tetrasporangia $; \mathrm{C}=$ cystocarps $; \mathrm{B}=$ both $\mathrm{S}=$ spermatangia $; \mathrm{M}=$ monospores; $\mathrm{O}=$ oogonia; $\mathrm{A}=$ antheridia; $\mathrm{b}=$ both; $\mathrm{P}=$ plurilocular and $\mathrm{U}=$ unilocular sporangia; $\mathrm{p}=$ propagules.)

Glen Maye Bay,

Stroin Vuigh, July 1959

\section{Off Charran, Sept. 1958}

Off Creg Liauyr Aug. Nov.
Depth (m below ELWS)

Cladophora sp.

Enteromorpha clathrata

(sensu Bliding, 1944)

Ulva lactuca

Acrothrix gracilis

Arthrocladia villosa

Chorda filum (sensu Hamel I93I-9)

Cladostephus spongiosus

(sensu Hamel r93 I-9)

Cutleria multifida gametophyte

C. multifida sporophyte

Desmarestia aculeata

Dictyota dichotoma

Ectocarpus confervoides

Halidrys siliquosa

Laminaria saccharina

Myriocladia? sp. (see Parke, I950)

Saccorhiza polyschides

Sphacelaria bipinnata

$S$. pennata

$S$. plumula

Sporochnus pedunculatus

Stictyosiphon tortilis

Antithamnion plumula

Bonnemaisonia asparagoides

Brongniartella byssoides

Callithamnion corymbosum

Ceramium rubrum agg.

Chylocladia verticillata

Corynospora pedicellata

Cryptopleura ramosa

Delesseria sanguinea

Halarachnion ligulatum

Heterosiphonia plumosa

Hypoglossum woodwardii

Lomentaria clavellosa

Membranoptera alata

Nitophyllum agg.

Odonthalia dentata

Phycodrys rubens

Plocamium coccineum

Polysiphonia brodiaei

$P$. elongata

$P$. urecolata agg.

$P$. violacea agg.

Pterosiphonia parasitica

P. thuyoides

Ptilota plumosa

Rhodomela confervoides

Scinaia furcellata

Sphondylothamnion multifidum

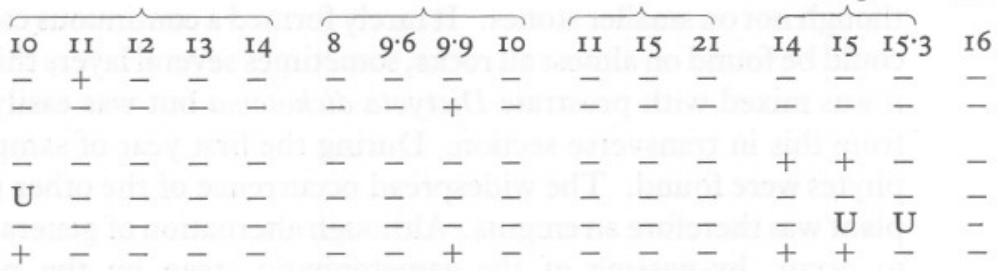

$+--\infty-\infty-\infty-\infty-$

$-+++$

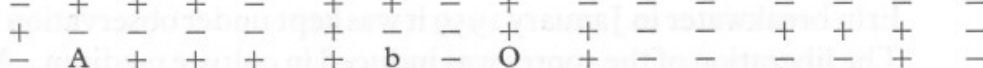

$+-\mathrm{P}-\mathrm{P}-\mathrm{P}-\mathrm{C}-\mathrm{-}-\mathrm{-}$

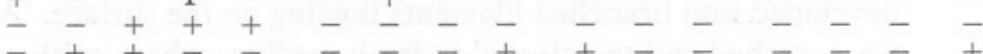

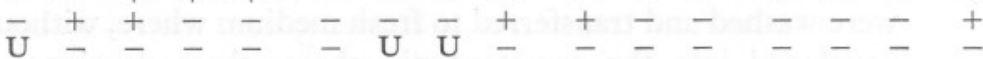

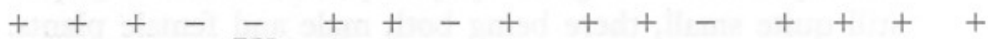

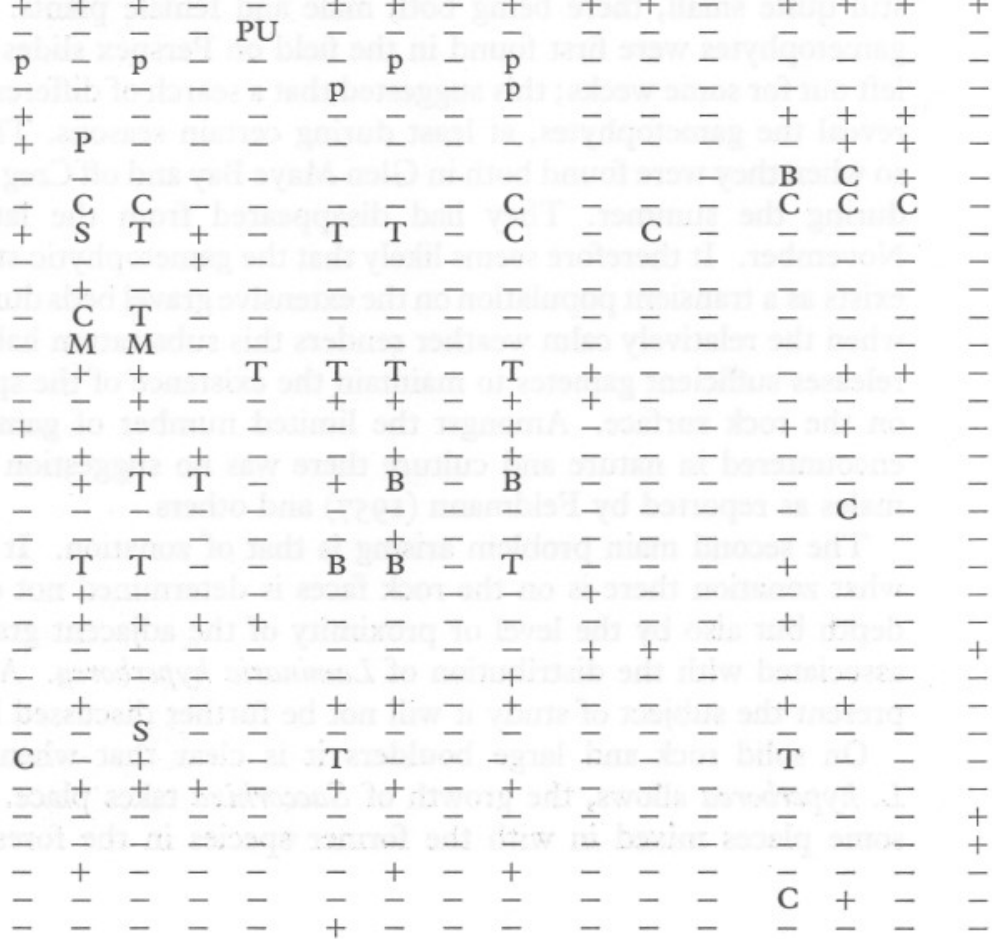

JOURN. MAR. BIOL. ASSOC. VOL. 39, I960 


\section{DISCUSSION}

The aim of a preliminary survey of this nature is the recognition of the ecological problems peculiar to the habitat. The first that arises here concerns what was undoubtedly the most ubiquitous sublittoral plant, the sporophyte of Cutleria multifida (Aglaozonia parvula). It occurred at virtually every level sampled in all the localities except Langness, on rocky bottoms and on boulders though not on smaller stones. It rarely formed a continuous carpet, but patches could be found on almost all rocks, sometimes several layers thick. Occasionally it was mixed with prostrate Dictyota dichotoma but was easily distinguishable from this in transverse section. During the first year of sampling no gametophytes were found. The widespread occurrence of the other generation of the plant was therefore an enigma. Although alternation of generations is supposed to occur, by-passing of the gametophytic stage by the production of an Aglaozonia-like phase by zoospores from the sporophyte has been reported (Church, I898). It was therefore thought likely that this might be the normal occurrence in these waters, and when a reproducing thallus was found on Port Erin breakwater in January 1959 it was kept under observation in the laboratory. The liberation of the spores was induced in culture medium. After 2 weeks they developed into branched filaments floating on the surface. A number of these were washed and transferred to fresh medium where, without exception, they developed into the gametophytic phase; these developed sporangia while still quite small, there being both male and female plants. Later that year gametophytes were first found in the field on Perspex slides which had been left out for some weeks; this suggested that a search of different habitats might reveal the gametophytes, at least during certain seasons. This proved to be so when they were found both in Glen Maye Bay and off Creg Liauyr (Table 4) during the summer. They had disappeared from the latter position by November. It therefore seems likely that the gametophytic stage of this plant exists as a transient population on the extensive gravel beds during the summer, when the relatively calm weather renders this substratum habitable, and then releases sufficient gametes to maintain the existence of the sporophytic phase on the rock surface. Amongst the limited number of gametophytic plants encountered in nature and culture there was no suggestion of a shortage of males as reported by Feldmann (1957) and others.

The second main problem arising is that of zonation. It is apparent that what zonation there is on the rock faces is determined not only by absolute depth but also by the level or proximity of the adjacent gravel bed. This is associated with the distribution of Laminaria hyperborea. As this plant is at present the subject of study it will not be further discussed here.

On solid rock and large boulders it is clear that when the absence of L. hyperborea allows, the growth of Saccorhiza takes place. It is present in some places mixed in with the former species in the forest, but it has its 
climax in the zone just below the Laminaria limit, where the light is evidently still sufficient for it but where the factor which limits Laminaria is evidently ineffective against it. It is probable that through its power of faster growth it can take advantage of transiently good conditions and become established, but because the plants are much less strongly constructed they last for a shorter time. It is worth stressing that the level of Saccorhiza dominance is dependent on the level of the Laminaria, at whatever depth this is.

The species forming the undergrowth of the Laminaria forest are not confined to that region and all extend downwards. The cover gradually becomes thinner, and near to the limit of the rock there are large patches of uninhabited surface. At the breakwater this bare zone is at the same depth as the rich forest flora of the Bowes or Spanish Head. This is in contrast to the situation in the Mediterranean, where Drach (1948a) insists that cover is always $100 \%$; where the rock is not occupied by plants it is colonized by animals. It seems that some factor associated with the proximity of the gravel, which inhibits the establishment of algae, also acts on the animals. Where light alone is the limiting factor, on overhangs and possibly the deeper rock at Spanish Head, the place of algae may be taken by Alcyonium and sponges.

The presence of a number of algae on the lowest known rock at the darkest time of the year makes the determination of the general lower algal limit for the area impossible.

Thirdly, there arises the question of the effect of depth on reproduction. Jones (1956) suggested that the greater paucity of fertile species at the greater depth of $25 \mathrm{~m}$ at Bardsey might reflect a weaker ability to reproduce nearer the limit of the range of a plant, but this cannot be supported by the present records. The reason might be that in most cases in the Isle of Man the lower limit of sublittoral algae is determined by a factor acting not on the growth rate (such as light) but on initial establishment (such as the proximity of the gravel). A second suggestion made by Jones was that carposporic plants of Brongniartella byssoides and Lomentaria clavellosa grew deeper than tetrasporic plants; this was however based on single samples. All the available records in the present work have been examined with this in mind, but only Brongniartella showed any indication of a relationship between reproductive phase and depth. With this species, as at Bardsey, gametophytic plants were confined to deeper water, below Io m, though there were only three records. There were 6 records of tetrasporic plants and only one of these was deeper than $10 \mathrm{~m}$. This lends support to Jones's evidence.

I am indebted to J. Faragher and L. Collister, crew of the motor-boat 'Cypris', and am particularly grateful to the latter for his unremitting vigilance at the other end of the safety line.

A number of species (though not all) were identified or checked by certain people; very many thanks for this are due to Dr H. Blackler (Ectocarpus, 
Giffordia), Dr E. M. Burrows (Enteromorpha, Tilopteris), Dr P. S. Dixon (Rhodophyceae), Dr D. E. G. Irvine (Sphacelaria), Dr M. W. Parke (Myriocladia?) and Dr M. Roberts (née Naylor) (Stictyosiphon).

I am also grateful to Miss W. B. Chapman for help with the maintenance of diving equipment, and would like to thank Professor W. T. Williams for helpful criticism of the manuscript.

\section{SUMMARY}

A general survey has been made of the distribution with depth of the main sublittoral algae inhabiting the rock surfaces at ten places around the south end of the Isle of Man, using an aqualung. The region is characterized by the lack of any deep water rock; this is replaced by gravel at a depth of about $12 \mathrm{~m}$ in most places. Immediately below ELWS the vegetation was dominated by Laminaria hyperborea, forming a thick forest with an undergrowth of smaller brown and red algae. The lower limit of this forest was well defined, and depended not on the absolute depth but on the proximity of the junction of solid rock and the gravel bed. Saccorhiza polyschides dominated just below the Laminaria hyperborea limit, at whatever depth this was. The smaller algae of the forest extended down on the deeper rock but the cover was reduced as the gravel was approached. Observations were made on one reef throughout the year. Although the abundance and form of the more important species varied, most were present at all times of the year, but Dictyota dichotoma was absent in winter. The deepest rock found, at $22 \mathrm{~m}$ below ELWS, was colonized by a few algae even in winter. The most ubiquitous plant on the rock was the sporophyte of Cutleria multifida which appeared to alternate with sexual plants which formed part of a transient summer population of algae which occupied the gravel bed in places.

\section{REFERENCES}

Admiralty Tide Tables, European Waters. London: Hydrographic Department, Admiralty.

AleEM, A. A., 1956. Quantitative underwater study of benthic communities inhabiting kelp beds off California. Science, Vol. 123, p. I83.

BLIDING, C., I944. Zur Systematik der schwedischen Enteromorphen. Bot. Notiser, I944, Hft. 3, pp. 33I-56.

BurRows, E. M., I958. Sublittoral algal population in Port Erin Bay, Isle of Man. F. mar. biol. Ass. U.K., Vol. 37, pp. 687-703.

BURSA, A., WojTUSIAK, H. \& WoJTUSIAK, R. J., I948. Investigations of the bottom fauna and flora in the Gulf of Gdansk by using a diving helmet. Bull. int. Acad. Cracovie (Acad. pol. Sci.), B. II, 1947, pp. 213-39.

Chapman, V. J. 1947. Methods of surveying Laminaria beds. F. mar. biol. Ass. U.K., Vol. 26, pp. 37-60.

ChURCH, A. H., I898. The polymorphy of Cutleria multifida (Grev.). Ann. Bot., Lond., Vol. 12, pp. 75-I09. 
DRACH, P., I948 $a$. Premières recherches en scaphandre autonome sur le peuplement des faciès rocheux de la zone littorale profonde. C.R. Acad. Sci., Paris, T. 227, pp. $1176-8$.

_ $1948 b$. Limite 'd expansion des peuplements benthiques sessiles en zone littorale profonde (faciès rocheux). C.R. Acad. Sci., Paris, T. 227, pp. 1397-9.

- 1949. Premières recherches en scaphandre autonome sur les formations de laminaires en zone littorale profonde. C.R. Soc. Biogéogr., T. 26, No. 227, pp. 46-9.

_ I95I. Les peuplements de fonds rocheux en zone littorale profonde (Recherches en scaphandre autonome). Année biol., Sér. 3, T. 27, pp. 503-I I.

ERNST, J., I 955 . Sur la végétation sous-marine de la Manche d'après de observations en scaphandre autonome. C.R. Acad. Sci., Paris, T. 24I, pp. I066-8.

FeldMANN, J., I957. La reproduction des algues marines dans ses rapports avec leur situation géographique. Année biol., Sér. 3, T. 33, pp. 49-56.

ForsteR, G. R., 1954. Preliminary note on a survey of Stoke Point rocks with selfcontained diving apparatus. F. mar. biol. Ass. U.K., Vol. 33, pp. 34I-4.

- 1955. Underwater observations on rocks of Stoke Point and Dartmouth. F. mar. biol. Ass. U.K., Vol. 34, pp. 197-9.

- 1958. Underwater observations on the fauna of shallow rocky areas in the neighbourhood of Plymouth. F. mar. biol. Ass. U.K., Vol. 37, pp. 473-82.

GisLÉN, T. 1930. Epibioses of the Gullmar Fjord. Skriftser. Kristinebergs Zoologiska Station 1877-1927, Nos. 3-4, 503 pp.

Hamel, G., I93I-9. Phéophycées de France. 43I pp. Paris.

IRvine, D. E. G., I956. Notes on the British species of the genus Sphacelaria Lyngb. Trans. bot. Soc. Edinb., Vol. 37, pp. 24-45.

JoNEs, W. E., I956. The littoral and sublittoral marine algae of Bardsey. Rep. Bardsey Observatory, 1955, pp. 40-51.

KITCHING, J. A., I937. Studies in sublittoral ecology. II. Recolonisation at the upper margin of the sublittoral region with a note on the denudation of Laminaria forests by storms. F. Ecol., Vol. 25, pp. 482-95.

I94I. Studies in sublittoral ecology. III. Laminaria forest on the west coast of Scotland; a study of zonation in relation to wave action and illumination. Biol. Bull., Woods Hole, Vol. 80, pp. 324-37.

Kitching, J. A., Macan, T. T. \& Gilson, H. C., I934. Studies in sublittoral ecology. I. A submarine gully in Wembury Bay, S. Devon. F. mar. biol. Ass. U.K., Vol. 19, pp. 677-705.

KNIGHT-JoNES, E. W. \& Clifford JoNes, W., I956. The fauna of rocks at various depths off Bardsey. I. Sponges, coelenterates and bryozoans. Rep. Bardsey Observatory, 1955, pp. 23-30.

LABOREL, J. \& VACELET, J., I958. Études des peuplements d'une grotte sousmarine du golfe de Marseille. Bull. Inst. océanogr. Monaco, No. I I20, 20 pp.

Lodge, S. M., I954. Sublittoral algal communities in the Isle of Man. Rapp. Comm. $8 m e$ Congr. int. bot., T. 17, p. 139.

NAYLOR, M., I958. Observations on the taxonomy of the genus Stictyosiphon Kütz. Rev. algol., T. 4, pp. 7-24.

PARKe, M., I950. Notes on the Plymouth marine flora. F. mar. biol. Ass. U.K., Vol. 29, pp. $257-61$.

_ I953. A preliminary check-list of British marine algae. F. mar. biol. Ass. U.K., Vol. 32, pp. 497-520.

- 1956. A preliminary check-list of British marine algae. Corrections and additions 1953-55. Brit. phycol. Bull., Vol. I, No. 4, pp. 26-31. 
PARKe, M., 1957. A preliminary check-list of British marine algae. Corrections and additions. II. 1956. Brit. phycol. Bull., Vol. I, No. 5, pp. 36-7.

PÉRÈs, J.-M. \& PICCARD, J., I949. Notes sommaires sur le peuplement des grottes sousmarines de la region de Marseille. C.R. Soc. Biogéogr., T. 26, No. 227, pp. 42-6.

StinN, D. J., 1959. Chemical constituents in sea water off Port Erin during 1958. Rep. mar. biol. Sta. Pt Erin, No. 7I, pp. 24-8.

SouthWARD, A. J., I953. The ecology of some rocky shores in the south of the Isle of Man. Proc. Lpool biol. Soc., Vol. 59, pp. I-50.

WaERN, M., I952. Rocky-shore algae in the Öregrund Archipelago. Acta phytogeogr. suec., Vol. 30, 298 pp.

WALKeR, F. T., I947. Sublittoral seaweed survey. I. Development of the view boxspring grab technique for sublittoral weed survey. II. Survey of Orkney, Scapa Flow. III. Survey of Orkney, Bay of Firth. F. Ecol., Vol. 35, pp. I66-85.

— 1950. Sublittoral seaweed survey of the Orkney Islands. F. Ecol., Vol. 38, pp. 139-65. 1954. A sublittoral survey of the Laminariaceae of Little Loch Broom. Trans. bot. Soc. Edinb., Vol. 36, pp. 305-8.

— 1957. An ecological study of Ailsa Craig, Holy Island, Inchmarnock, May Island and Seaforth Island. Trans. bot. Soc. Edinb., Vol. 37, pp. I82-99.

ZALOKAR, M., I942. Les associations sous-marines de la côte adriatique au dessous de Velabit. Bull. Soc. bot. Genève, T. 33, pp. 172-95. 